\title{
Violation of a Bell-like inequality by a combination of Rayleigh scattering with a Mach-Zehnder setup
}

\author{
Tom Rother \\ German Aerospace Center, Remote Sensing Technology Institute, D-17235 Neustrelitz, Germany
}

\section{A R T I C L E I N F O}

\section{Article history:}

Received 4 August 2015

Received in revised form

9 September 2015

Accepted 9 September 2015

\section{Keywords:}

Bell-like inequality

CHSH-inequality

Rayleigh scattering

Mach-Zehnder interferometry

\begin{abstract}
A B S T R A C T
In this paper I propose a classical optics experiment that results in a maximum violation of a Bell-like inequality. The first part is concerned with the Bell-like inequality (the so-called CHSH-inequality) itself. Its importance and its maximum violation in Quantum Mechanics $(\mathrm{QM})$ are discussed in detail by employing an abstract probability state concept in a 4-dim. but classical event space. A T-matrix that represents the integral part of a corresponding Green's function as well as a statistical operator that contains a negative quasi-probability can be related to the corresponding quantum mechanical experiment. It is demonstrated that the derivation and usage of the T-matrix and the Green's function is equivalent to what is known from classical scattering theory. It is shown moreover that the negative quasi-probability of the statistical operator may be interpreted as a sink of probabilities related to two single events of the considered 4-dim. event space. A necessary condition for the violation of the $\mathrm{CHSH}$-inequality is derived and discussed afterwards. In the second part of this paper I discuss a modification of the 4-dim. event space considered in the first part. It is shown that a combination of conventional Rayleigh scattering with a MachZehnder setup would be able to put this modification into practice. Thus it becomes possible to achieve a maximum violation of the $\mathrm{CHSH}$-inequality, if formulated in terms of intensities, on a pure classical way. The combination of classical light scattering with correlation experiments such as proposed in this paper may open new ways to study and to use the violation of Bell-like inequalities in modern optics.
\end{abstract}

(c) 2015 Elsevier Ltd. All rights reserved.

\section{Introduction}

In 1964 J.S. Bell published his famous paper regarding the just as well famous Einstein-Podolsky-Rosen (EPR) paradox - a paradox that had been discussed since 1935 until Bell's paper purely on a philosophical level [1]. EPR is concerned with the question if QM can be considered to be a complete theory, or if we have to search for any hidden parameters to complete it [2,3]. In his paper Bell derived an inequality, formulated in terms of correlations, for a certain class of experiments. He proved that this inequality cannot be violated by any of such experiments or any theory of local realism. The term "local" in this context expresses the fact that there is no "spooky" action at a distance between two distant measurement locations. And that a measurement is "realistic" means that the property of an object we intend to measure can be related to this object independent of whether we perform the measurement or not. Or, in other words: "The moon is there even when not being observed". Today it is tacitly assumed that any classical theory (Newtonian mechanic, Electrodynamic, etc.) belong to a theory of local realism. And, moreover, the impossibility of any such theory to violate Bell's inequality holds independent of whether there exist hidden parameters or not. 5 years later, in 1969, J. Clauser, M. Horne, A. Shimony, and R. Holt published an alternative inequality - the so-called CHSH-inequality [4]. Since then this inequality has become a much-cited relation. A first experiment to demonstrate its violation in QM was put into practice in 1982 by A. Aspect and co-workers. They 
used polarization entangled photons to create a 4-dim. event space [5]. Several other quantum mechanical experiments have been developed in the meantime. They are of growing importance not only for questions regarding the foundations of QM but also in quantum information theory (e.g., quantum cryptography and quantum computing), and in quantum optics. Today, the violation of the CHSH-inequality in QM is accepted among most of the physicists as an experimental fact. It is assumed that this violation expresses the non-local character of this theory, and, more important, that it is exactly this non-local character that makes QM essentially differ from our classical lines of thought. The following two representative quotations are meant to emphasize this point of view:

Bell's theorem, which states that the prediction of quantum theory cannot be accounted for by any local theory, represents one of the most profound developments in the foundation of physics [6].

Because quantum mechanics violates Bell's inequality, it is in empirical disagreement with the family of local physical theories [7]

These and similar quotations reflect the position that the $\mathrm{CHSH}$-inequality provides us with a bound of any related correlation experiment if performed with a classical setup. However, there is still an ongoing discussion regarding the possibility to violate Bell's inequality with a classical experiment. In 2010 Borges et al. performed a classical optics experiment that results in a maximum violation of a Bell-like (or CHSH-like) inequality if formulated in terms of intensities [8]. It is based on nonseparable spin-orbit modes of a laser beam. In what follows I propose an alternative classical optics experiment that will also result in a maximum violation of the $\mathrm{CHSH}-$ inequality.

In the first part of this paper the CHSH-inequality and its maximum violation by a corresponding quantum mechanical experiment are discussed. An abstract probability state concept in a 4-dim. but classical event space is introduced to this end. Using a T-matrix will allow us to describe the experiment in terms of a basis transformation, as it is known from classical scattering theory [11]. It turns out that this T-matrix represents the decisive element of the interaction part of a corresponding Green's function. The existence of two sets of different basis vectors can be considered to be the result of two local and independent interaction mechanisms described on the level of the initially introduced abstract probability states. A statistical operator with negative quasi-probabilities can moreover be related to this experiment. The existence and meaning of such negative probabilities are other aspects of ongoing discussions in $\mathrm{QM}[9,10]$. Here it is shown that the negative quasi-probabilities may be considered as a redistribution of (always positive) probabilities related to each single event of the 4-dim. event space. A necessary condition for the violation of the CHSH-inequality is derived and discussed afterwards. It is used as a proof that the classical counterpart of the quantum mechanical experiment will never be able to violate the $\mathrm{CHSH}$-inequality.

The second part of this paper is concerned with the description of a classical optics experiment that will result in a violation of the CHSH-inequality if formulated in terms of intensities. For this we have to discuss an appropriate modification of the 4-dim. event space presented in the first part of this paper. It is finally shown that a combination of conventional Rayleigh scattering with a Mach-Zehnder setup would be able to accomplish this modification on a pure classical way. Thus it is demonstrated that it is questionable to relate the violation of Belllike inequalities exclusively to the realm of QM without any reference to the experimental context. The violation of Bell-like inequalities with such classical experiments is moreover an indication that we have to revisit our understanding of "non-local theories". However, classical correlation experiments such as performed in [8] or proposed in this paper may open new ways to study and to use the violation of Bell-like inequalities in modern optics. Correlation measurements can be applied in a similar manner as known from scattering experiments, for example. i.e., the deviation from an initially given correlation or a combination of correlations can be used to gain information about the disturbance that causes this deviation. The CHSH-inequality - especially if used with parameter sets resulting in a maximum violation - is just one example that provides us with such an initial combination of correlations.

\section{CHSH-Inequality and its violation in QM}

\subsection{The quantum mechanical Bell's box and the 4-dim. but classical event space}

Let us start with a quite phenomenological description of a quantum mechanical Bell's experiment by introducing a "quantum mechanical Bell's box" (QBB). In its first level of configuration our QBB consists of a box with three compartments (see Fig. 1). In the center compartment we have placed a source that emits two polarization entangled photons (horizontally $(h)$ - and vertically $(v)$-polarized with respect to a fixed but arbitrary plane) into opposite directions once we push the button. But we do not know the state of polarization of the photon emitted in a certain direction. i.e., we do not know if we have the combination $(h, v)$ or $(v, h)$ with respect to the polarization of both photons in a single event. The first term in the brackets is related to the photon on the left-hand side, and the second term is related to the photon on the right-hand side. It should be emphasized that the $h$-or $v$-polarization represents the state of a photon but not the measurement

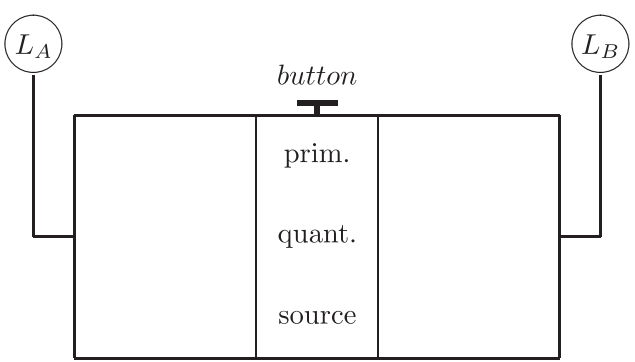

Fig. 1. Bell's box with only a primary quantum mechanical source. 
value! This is sometimes confused in the literature even though it is well-known already from classical electrodynamics. For the time being, the other two compartments on the left- and right-hand side remain empty. A lamp is mounted at each end of the box (lamp $L_{A}$ and $L_{B}$ ) and equipped with a detector that switches the lamp on if a $h$ polarized photon is detected. The lamp remains switched off otherwise. Thus we have the two measurement values "yes" (lamp switched on) and "no" (lamp switched of) in this experiment. There are in general 4 non-local measurement pairs that establish a 4-dim. but classical event space. These are "lamp $L_{A}$ on, lamp $L_{B}$ off: $\left(y_{A}, n_{B}\right)$ ", "lamp $L_{A}$ off, lamp $L_{B}$ on: $\left(n_{A}, y_{B}\right)$, "both lamps on: $\left(y_{A}, y_{B}\right)$ ", and "both lamps off: $\left(n_{A}, n_{B}\right)$ ". After performing a multitude of measurements with this first level setup of the QBB we realize that there are just two possible events. These are $\left(y_{A}, n_{B}\right)$ and $\left(n_{A}, y_{B}\right)$. Let us further assume that the primary stochastic source is prepared in such a way that each of the two possible pairs of photons is emitted with the probability of $1 / 2$. Employing an abstract probability state concept, this experiment and its outcome can be described in the following way:

If we relate the eigenvector

$\left|\varphi_{1}\right\rangle=(1,0)$

to the local measurement value "lamp A/B on: $\left(y_{A / B}\right)$ ", and the eigenvector

$\left|\varphi_{2}\right\rangle=(0,1)$

to the local measurement value "lamp A/B off: $\left(n_{A / B}\right)$ ", then we are able to characterize this first level QBB by the abstract probability state

$\left|\Phi_{Q B B}^{(0)}\right\rangle=\frac{1}{\sqrt{2}} \cdot\left(\left|\varphi_{1}, \varphi_{2}\right\rangle-\left|\varphi_{2}, \varphi_{1}\right\rangle\right)$

in the direct product space. Then, the scalar products

$\left\langle\Phi_{y, n} \mid \Phi_{y, n}\right\rangle=p(y, n)$

$\left\langle\Phi_{n, y} \mid \Phi_{n, y}\right\rangle=p(n, y)$

of the projections

$\left|\Phi_{y, n}\right\rangle=\left|\varphi_{1}, \varphi_{2}\right\rangle \cdot\left\langle\varphi_{2}, \varphi_{1} \mid \Phi_{Q B B}^{(0)}\right\rangle$

$\left|\Phi_{n, y}\right\rangle=\left|\varphi_{2}, \varphi_{1}\right\rangle \cdot\left\langle\varphi_{1}, \varphi_{2} \mid \Phi_{\mathrm{QBB}}^{(0)}\right\rangle$

provide us with the measured probabilities $p(y, n)=$ $p(n, y)=1 / 2$ of the two possible measurement values $\left(y_{A}, n_{B}\right)$ and $\left(n_{A}, y_{B}\right)$. Note that the scalar product of the two vectors $|f, g\rangle$ and $|p, q\rangle$ in the direct product space is defined according to

$\langle g, f \mid p, q\rangle:=\langle f \mid p\rangle \cdot\langle g \mid q\rangle$.

In a second level of configuration we insert two additional interaction modules into the so far empty compartments of the QBB. These modules are nothing but polarization filters the photons on both sides are interacting with. They are located at $x=+x_{w}$ and $x=-x_{w}$ on the right- and left-hand side from the primary source located at $x=0$.The orientation of the polarization filters with respect to a fixed plane can be continuously varied between $[0, \pi]$ with corresponding rotating switches $D_{\alpha}$ and $D_{\beta}$. The local interactions become in this way

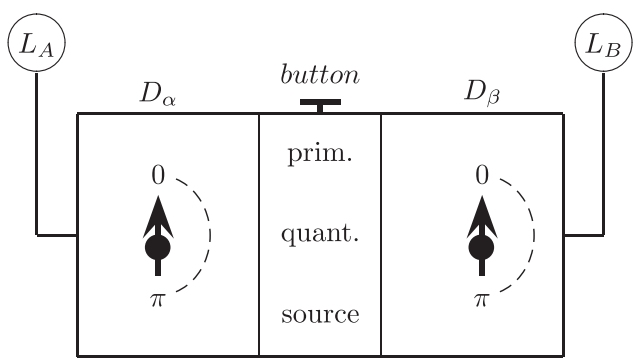

Fig. 2. Bell's box with the primary quantum mechanical source of Fig. 1 but now with two additional, local stochastic interaction modules (polarization filters).

functions of the local parameters $\alpha$ and $\beta$. Finally, the detectors of the lamps are replaced by new detectors which act in such a way that the lamps are switched on if a photon is detected, independent of its state of polarization. The lamps remain switched off otherwise. This second level configuration of our Bell's experiment is depicted in Fig. 2. Now, all 4 possible measurement pairs can be observed. Let us further assume that performing a large number of experiments with different sets of the local parameters $\alpha$ and $\beta$ result in the following probabilities:

- probability $p(y, y) / p(n, n)$ that both lamps are switched on/switched off:

$$
p(y, y)=p(n, n)=\frac{1}{2} \cdot \sin ^{2}(\alpha-\beta)
$$

- probability $p(y, n) / p(n, y)$ that just one lamp is switched on and the other lamp remains switched off:

$$
p(n, y)=p(y, n)=\frac{1}{2} \cdot \cos ^{2}(\alpha-\beta)
$$

These probabilities can indeed be observed in corresponding quantum mechanical experiments and can therefore accepted to be a fact. It allows us to relate the following abstract probability state to this second level QBB:

$$
\begin{aligned}
\left|\Phi_{\mathrm{QBB}}\right\rangle= & c_{11} \cdot\left|\varphi_{1}, \varphi_{1}\right\rangle+c_{12} \cdot\left|\varphi_{1}, \varphi_{2}\right\rangle \\
& +c_{21} \cdot\left|\varphi_{2}, \varphi_{1}\right\rangle+c_{22} \cdot\left|\varphi_{2}, \varphi_{2}\right\rangle,
\end{aligned}
$$

with the $\alpha$ and $\beta$ dependent probability amplitudes

$c_{11}=c_{22}=\frac{1}{\sqrt{2}} \cdot \sin (\alpha-\beta)$

$c_{12}=-c_{21}=\frac{1}{\sqrt{2}} \cdot \cos (\alpha-\beta)$.

The additional eigenvectors $\left|\varphi_{1}, \varphi_{1}\right\rangle$ and $\left|\varphi_{2}, \varphi_{2}\right\rangle$ are related to the two additional measurement pairs $\left(y_{A}, y_{B}\right)$ and $\left(n_{A}, n_{B}\right)$. If $\alpha=\beta$ is chosen, then we end up with (3) as a special case. This probability state is normalized to unity, i.e., we have

$\left\langle\Phi_{\mathrm{QBB}} \mid \Phi_{\mathrm{QBB}}\right\rangle=1$.

By the way, the same normalization holds obviously for the probability state (3). The probabilities (9) and (10) can 
then be calculated from the scalar products of the corresponding projections

$$
\begin{aligned}
& \left|\Phi_{y, n}\right\rangle=\left|\varphi_{1}, \varphi_{2}\right\rangle \cdot\left\langle\varphi_{2}, \varphi_{1} \mid \Phi_{\mathrm{QBB}}\right\rangle \\
& \left|\Phi_{n, y}\right\rangle=\left|\varphi_{2}, \varphi_{1}\right\rangle \cdot\left\langle\varphi_{1}, \varphi_{2} \mid \Phi_{\mathrm{QBB}}\right\rangle \\
& \left|\Phi_{y, y}\right\rangle=\left|\varphi_{1}, \varphi_{1}\right\rangle \cdot\left\langle\varphi_{1}, \varphi_{1} \mid \Phi_{\mathrm{QBB}}\right\rangle \\
& \left|\Phi_{n, n}\right\rangle=\left|\varphi_{2}, \varphi_{2}\right\rangle \cdot\left\langle\varphi_{2}, \varphi_{2} \mid \Phi_{\mathrm{QBB}}\right\rangle .
\end{aligned}
$$

\subsection{T-matrix, Green's function, and statistical operator of the $\mathrm{QBB}$ experiment}

Fig. 3 shows the general scheme that can be used to describe this second level QBB experiment in terms of two local interactions. $h$ represents a horizontally polarized photon emitted from the primary source into a certain direction. Correspondingly, $v$ represents a vertically polarized photon emitted by the same source and at the same time but into the opposite direction. The local events on each side are $\left(n_{A / B}\right)$ ("lamp A/B off") and $\left(y_{A / B}\right)$ ("lamp A/ $\mathrm{B}$ on"). Eigenvector $\left|\varphi_{1}\right\rangle$ is again related to $y_{A / B}$, and eigenvector $\left|\varphi_{2}\right\rangle$ to $n_{A / B}$. We are now interested in a justification of the different probabilities of the 4 possible event pairs $\left(y_{A}, y_{B}\right),\left(y_{A}, n_{B}\right),\left(n_{A}, y_{B}\right)$, and $\left(n_{A}, n_{B}\right)$. The two substates before the additional interactions are given according to (3) by

$\left|\Phi_{1}^{(0)}\right\rangle=\frac{1}{\sqrt{2}} \cdot\left|\varphi_{1}, \varphi_{2}\right\rangle$

and

$\left|\Phi_{2}^{(0)}\right\rangle=-\frac{1}{\sqrt{2}} \cdot\left|\varphi_{2}, \varphi_{1}\right\rangle$.

The two local stochastic interactions on each side and the resulting local probability amplitudes (the sine and cosine functions in the square brackets in Fig. 3) may be obtained by use of two local and unitary T-matrices which are identical with the matrix of rotation,

$\mathbf{T}_{\boldsymbol{\alpha} / \boldsymbol{\beta}}=\mathbf{D}_{\boldsymbol{\alpha} / \boldsymbol{\beta}}=\left(\begin{array}{cc}\cos \alpha / \beta & -\sin \alpha / \beta \\ \sin \alpha / \beta & \cos \alpha / \beta\end{array}\right)$.

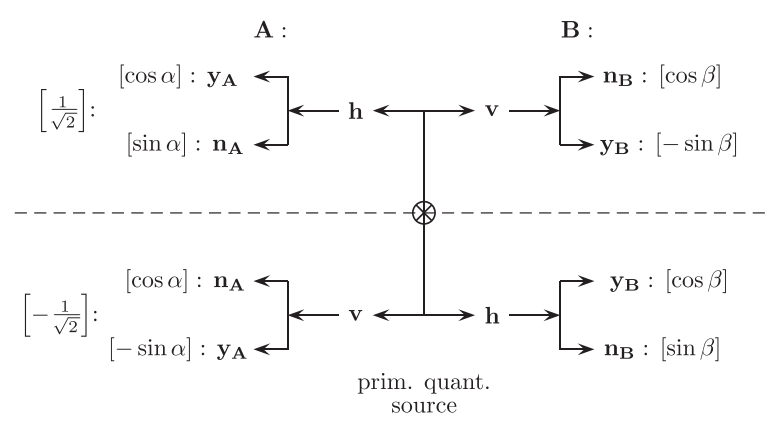

Fig. 3. Interaction scheme of Bell's experiment. $h$ and $v$ are the possible states of polarization of the photons emitted by the primary source. $n_{A / B}$ ("lamp A/B off") and $y_{A / B}$ ("lamp A/B on") are the local events measured on each side in the experiment after the additional local interactions took place. The corresponding local probability amplitudes are given in the square brackets.
The amplitudes $c_{1^{\prime}}$ and $c_{2^{\prime}}$ of the local probability states after the interaction are then the result of relation

$$
\left(\begin{array}{l}
c_{1^{\prime}} \\
c_{2^{\prime}}
\end{array}\right)=\mathbf{T}_{\boldsymbol{\alpha} / \boldsymbol{\beta}} \cdot\left(\begin{array}{l}
c_{1} \\
c_{2}
\end{array}\right)
$$

with $c_{1}$ and $c_{2}$ being the amplitudes of the local probability states before the interaction (i.e., $c_{2}=-c_{1}=1 / \sqrt{2}$, according to (19) and (20)). This description is identical with the description of the classical interaction of a linearly polarized plane wave with a polarization filter rotated by an angle of $\alpha$ (or $\beta$ ) against the plane of linear polarization. After the interaction, from (22) and the respective T-matrices we get therefore the following local probability states of the 4 two-dim. subspaces:

- local observation point $A$ and $h$-polarization:

$$
|\phi(A, h)\rangle=\frac{1}{\sqrt{2}} \cdot\left(\cos \alpha \cdot\left|\varphi_{1}\right\rangle+\sin \alpha \cdot\left|\varphi_{2}\right\rangle\right)
$$

- local observation point $A$ and $v$-polarization:

$$
|\phi(A, v)\rangle=-\frac{1}{\sqrt{2}} \cdot\left(-\sin \alpha \cdot\left|\varphi_{1}\right\rangle+\cos \alpha \cdot\left|\varphi_{2}\right\rangle\right)
$$

- local observation point $B$ and $h$-polarization:

$$
|\phi(B, h)\rangle=\cos \beta \cdot\left|\varphi_{1}\right\rangle+\sin \beta \cdot\left|\varphi_{2}\right\rangle
$$

- local observation point $B$ and $v$-polarization:

$$
|\phi(B, v)\rangle=-\sin \beta \cdot\left|\varphi_{1}\right\rangle+\cos \beta \cdot\left|\varphi_{2}\right\rangle .
$$

Please, note that we have assigned the probability amplitudes of the primary source to the local probability states on the left-hand side. In the next step we pass on from these local substates to the direct product states that belong to the event pairs of both sides. This procedure is accomplished separately for the upper and lower part of Fig. 3. That is because these parts belong to successive time steps in our experiment. In doing so, we get the following two probability states in the direct product space:

$$
\begin{aligned}
\left|\Phi_{1}\right\rangle=|\phi(A, h)\rangle \otimes|\phi(B, v)\rangle=\frac{1}{\sqrt{2}} \\
\cdot\left[-\cos \alpha \cdot \sin \beta \cdot\left|\varphi_{1}, \varphi_{1}\right\rangle+\cos \alpha \cdot \cos \beta \cdot\left|\varphi_{1}, \varphi_{2}\right\rangle\right. \\
\left.-\sin \alpha \cdot \sin \beta \cdot\left|\varphi_{2}, \varphi_{1}\right\rangle+\sin \alpha \cdot \cos \beta \cdot\left|\varphi_{2}, \varphi_{2}\right\rangle\right]
\end{aligned}
$$

(this state belongs to the upper part of Fig. 3), and

$$
\begin{aligned}
& \left|\Phi_{2}\right\rangle=|\phi(A, v)\rangle \otimes|\phi(B, h)\rangle=\frac{1}{\sqrt{2}} \\
& \quad \cdot\left[\sin \alpha \cdot \cos \beta \cdot\left|\varphi_{1}, \varphi_{1}\right\rangle+\sin \alpha \cdot \sin \beta \cdot\left|\varphi_{1}, \varphi_{2}\right\rangle\right. \\
& \left.\quad-\cos \alpha \cdot \cos \beta \cdot\left|\varphi_{2}, \varphi_{1}\right\rangle-\cos \alpha \cdot \sin \beta \cdot\left|\varphi_{2}, \varphi_{2}\right\rangle\right]
\end{aligned}
$$

(this state belongs to the lower part of Fig. 3). Now it is straightforward to see that the superposition of both substates results indeed into state (11) with amplitudes (12) and (13). The same experiment can be described from 
the alternative point of view of a basis transformation, as we will demonstrate now.

We ask for the transformation matrix that transforms the primary probability state (3) into the new probability state

$$
\begin{aligned}
\left|\Phi_{Q B B}\right\rangle= & c_{y y} \cdot\left|y_{A}, y_{B}\right\rangle+c_{y n} \cdot\left|y_{A}, n_{B}\right\rangle \\
& +c_{n y} \cdot\left|n_{A}, y_{B}\right\rangle+c_{n n} \cdot\left|n_{A}, n_{B}\right\rangle
\end{aligned}
$$

with probability amplitudes

$c_{y y}=c_{n n}=\frac{1}{\sqrt{2}} \cdot \sin (\alpha-\beta)$

$c_{y n}=-c_{n y}=\frac{1}{\sqrt{2}} \cdot \cos (\alpha-\beta)$

The new but so far unknown eigenvectors $\left|y_{A / B}\right\rangle$ and $\left|n_{A / B}\right\rangle$ are again related to the possible local measurements "lamp A/B on" and "lamp A/B off". Now, let us assume that these eigenvectors are the result of a rotation of the local coordinate system on the left- and right-hand side,

$\left(\begin{array}{c}\left|y_{A / B}\right\rangle \\ \left|n_{A / B}\right\rangle\end{array}\right)=\mathbf{D}_{\alpha / \beta} \cdot\left(\begin{array}{c}\left|\varphi_{1}\right\rangle \\ \left|\varphi_{2}\right\rangle\end{array}\right)$,

caused by the two additional polarization filters. $\mathbf{D}_{\boldsymbol{\alpha} / \boldsymbol{\beta}}$ therein is again the matrix (21) of rotation. Thus we get

$\left|y_{A}\right\rangle=(\cos \alpha,-\sin \alpha)$

$\left|y_{B}\right\rangle=(\cos \beta,-\sin \beta)$

$\left|n_{A}\right\rangle=(\sin \alpha, \cos \alpha)$

$\left|n_{B}\right\rangle=(\sin \beta, \cos \beta)$.

These 4 vectors are orthonormal among each other, i.e., they form a new basis in the considered 4-dim. direct product space. Next, we introduce the T-matrix

$\begin{aligned} \mathbf{T} & =\left(\begin{array}{cccc}\left\langle y_{B}, y_{A} \mid \varphi_{1}, \varphi_{1}\right\rangle & \left\langle y_{B}, y_{A} \mid \varphi_{1}, \varphi_{2}\right\rangle & \left\langle y_{B}, y_{A} \mid \varphi_{2}, \varphi_{1}\right\rangle & \left\langle y_{B}, y_{A} \mid \varphi_{2}, \varphi_{2}\right\rangle \\ \left\langle n_{B}, y_{A} \mid \varphi_{1}, \varphi_{1}\right\rangle & \left\langle n_{B}, y_{A} \mid \varphi_{1}, \varphi_{2}\right\rangle & \left\langle n_{B}, y_{A} \mid \varphi_{2}, \varphi_{1}\right\rangle & \left\langle n_{B}, y_{A} \mid \varphi_{2}, \varphi_{2}\right\rangle \\ \left\langle y_{B}, n_{A} \mid \varphi_{1}, \varphi_{1}\right\rangle & \left\langle y_{B}, n_{A} \mid \varphi_{1}, \varphi_{2}\right\rangle & \left\langle y_{B}, n_{A} \mid \varphi_{2}, \varphi_{1}\right\rangle & \left\langle y_{B}, n_{A} \mid \varphi_{2}, \varphi_{2}\right\rangle \\ \left\langle n_{B}, n_{A} \mid \varphi_{1}, \varphi_{1}\right\rangle & \left\langle n_{B}, n_{A} \mid \varphi_{1}, \varphi_{2}\right\rangle & \left\langle n_{B}, n_{A} \mid \varphi_{2}, \varphi_{1}\right\rangle & \left\langle n_{B}, n_{A} \mid \varphi_{2}, \varphi_{2}\right\rangle\end{array}\right) \\ = & \left(\begin{array}{cccc}\cos \alpha \cdot \cos \beta & -\cos \alpha \cdot \sin \beta & -\sin \alpha \cdot \cos \beta & \sin \alpha \cdot \sin \beta \\ \cos \alpha \cdot \sin \beta & \cos \alpha \cdot \cos \beta & -\sin \alpha \cdot \sin \beta & -\sin \alpha \cdot \cos \beta \\ \sin \alpha \cdot \cos \beta & -\sin \alpha \cdot \sin \beta & \cos \alpha \cdot \cos \beta & -\cos \alpha \cdot \sin \beta \\ \sin \alpha \cdot \sin \beta & \sin \alpha \cdot \cos \beta & \cos \alpha \cdot \sin \beta & \cos \alpha \cdot \cos \beta\end{array}\right)\end{aligned}$

The new probability amplitudes (30) and (31) may then be calculated from the primary probability amplitudes of representation (3) by use of the relation

$\left(\begin{array}{c}c_{y y} \\ c_{y n} \\ c_{n y} \\ c_{n n}\end{array}\right)=\mathbf{T} \cdot\left(\begin{array}{c}0 \\ 1 / \sqrt{2} \\ -1 / \sqrt{2} \\ 0\end{array}\right)$.

The T-matrix (37) is again a unitary matrix, i.e.,

$\mathbf{T}^{t p} \cdot \mathbf{T}=\mathbf{E}$

holds with $\mathbf{E}$ representing the $4 \times 4$ unit matrix. This property ensures the conservation of the overall probability for this transformation process. Such a representation of the T-matrix in terms of scalar products of the new and old basis vectors is already known from conventional scattering theory. Regarding Mie scattering, for example, the expansion of the primary incident plane wave in terms of regular wave functions is transformed at the surface of the sphere into an expansion in terms of outgoing wave functions. This transformation results in a similar T-matrix. Both the regular and outgoing wave functions form also two different basis systems at the surface of the sphere (see [11, Chapter 2], for example).

Now, it will be shown that the T-matrix (37) represents the decisive element of the interaction part of a Green's function that can be related to the quantum mechanical Bell's experiment. It is moreover demonstrated that this Green's function can be determined in close analogy to what is known from classical wave scattering (see [11], Chapter 4 therein, for example). For this purpose we consider once again the two different sets of basis vectors in the direct product space that is related to our 4-dim. event space. For simplicity, let us abbreviate these basis vectors by

$$
\begin{array}{ll}
\left|\Xi_{1}\right\rangle:=\left|\varphi_{1}, \varphi_{1}\right\rangle, & \left|\Xi_{2}\right\rangle:=\left|\varphi_{1}, \varphi_{2}\right\rangle \\
\left|\Xi_{3}\right\rangle:=\left|\varphi_{2}, \varphi_{1}\right\rangle, & \left|\Xi_{4}\right\rangle:=\left|\varphi_{2}, \varphi_{2}\right\rangle,
\end{array}
$$

and

$\begin{array}{ll}\left|\Psi_{1}\right\rangle:=\left|y_{A}, y_{B}\right\rangle, & \left|\Psi_{2}\right\rangle:=\left|y_{A}, n_{B}\right\rangle \\ \left|\Psi_{3}\right\rangle:=\left|n_{A}, y_{B}\right\rangle, & \left|\Psi_{4}\right\rangle:=\left|n_{A}, n_{B}\right\rangle .\end{array}$

The two expansions

$\mathbf{D}_{\mathbf{1}}=\sum_{i=1}^{4}\left|\boldsymbol{\Xi}_{i}\right\rangle\left\langle\boldsymbol{\Xi}_{i}\right|$

and

$\mathbf{D}_{\mathbf{2}}=\sum_{i=1}^{4}\left|\Psi_{i}\right\rangle\left\langle\Psi_{i}\right|$

in terms of dyadic products represent corresponding unit operators in the considered direct product space. Next, let us choose the following "ansatz" for the Green's function:

$$
\begin{aligned}
\mathbf{G}_{\mathbf{B}}= & \mathbf{G}_{\mathbf{B}}^{\mathbf{0}}+\mathbf{G}_{\mathbf{B}}^{\mathbf{W}}=\mathbf{D}_{\mathbf{1}} \cdot H\left(\left|x_{w}\right|-|x|\right) \\
& +\mathbf{D}_{\mathbf{2}} \mathbf{W}_{\mathbf{B}} \mathbf{D}_{\mathbf{1}} \cdot H\left(|x|-\left|x_{w}\right|\right) .
\end{aligned}
$$

$H(\cdot)$ therein denotes the Heaviside function, and $\mathbf{W}_{\mathbf{B}}$ represents the so far unknown interaction operator. $+x_{w}$ and $-x_{w}$ denote the distance of the additional polarization filters on the right- and left-hand side from the primary source located at $x=0$. The two parts of the Green's function are thus describing the situation in front and behind these interactions. Now, if applying one of the two additional conditions

$$
\left[\sum_{i=1}^{4}\left\langle\Xi_{i}\left|\mathbf{G}_{\mathbf{B}}\right| \boldsymbol{\Xi}_{i}\right\rangle\right]_{|x|<\left|x_{w}\right|}=\left[\sum_{i=1}^{4}\left\langle\boldsymbol{\Xi}_{i}\left|\mathbf{G}_{\mathbf{B}}\right| \boldsymbol{\Xi}_{i}\right\rangle\right]_{|x|>\left|x_{w}\right|}
$$

and

$$
\left[\sum_{i=1}^{4}\left\langle\Psi_{i}\left|\mathbf{G}_{\mathbf{B}}\right| \Psi_{i}\right\rangle\right]_{|x|<\left|x_{w}\right|}=\left[\sum_{i=1}^{4}\left\langle\Psi_{i}\left|\mathbf{G}_{\mathbf{B}}\right| \Psi_{i}\right\rangle\right]_{|x|>\left|x_{w}\right|}
$$

we are able to determine the interaction operator $\mathbf{W}_{\mathbf{B}}$ in a straightforward way. Using (45), for example, we get from 
(42) to (44)

$\sum_{i, k=1}^{4}\left\langle\boldsymbol{\Xi}_{i} \mid \boldsymbol{\Xi}_{k}\right\rangle\left\langle\boldsymbol{\Xi}_{k} \mid \boldsymbol{\Xi}_{i}\right\rangle=\sum_{i, k, l=1}^{4}\left\langle\boldsymbol{\Xi}_{i} \mid \Psi_{k}\right\rangle\left\langle\Psi_{k}\left|\mathbf{W}_{\mathbf{B}}\right| \boldsymbol{\Xi}_{l}\right\rangle\left\langle\boldsymbol{\Xi}_{l} \mid \boldsymbol{\Xi}_{i}\right\rangle$

With the definition

$\left[W_{B}\right]_{k l}:=\left\langle\Psi_{k}\left|\mathbf{W}_{\mathbf{B}}\right| \Xi_{l}\right\rangle, \quad k, l=1, \ldots, 4$

of the matrix elements of the interaction operator $\mathbf{W}_{\mathbf{B}}$ the right-hand side of (47) may be rewritten into

$\sum_{i, k, l=1}^{4}\left\langle\Xi_{i} \mid \Psi_{k}\right\rangle\left[W_{B}\right]_{k l}\left\langle\Xi_{l} \mid \Xi_{i}\right\rangle$

Next, we take advantage of the fact that the transformation between the two sets of basis vectors is accomplished by relation

$\left(\left|\Xi_{1}\right\rangle,\left|\Xi_{2}\right\rangle,\left|\Xi_{3}\right\rangle,\left|\Xi_{4}\right\rangle\right)=\left(\left|\Psi_{1}\right\rangle,\left|\Psi_{2}\right\rangle,\left|\Psi_{3}\right\rangle,\left|\Psi_{4}\right\rangle\right) \cdot \mathbf{T}$

with $\mathbf{T}$ according to (37) (note that the basis vectors in relation (50) are the elements of a "supervector"). Now, if identifying the matrix elements $\left[W_{B}\right]_{k l}$ in (49) with the elements of the T-Matrix (37) the right-hand side of (47) reads

$\sum_{i, l=1}^{4}\left\langle\boldsymbol{\Xi}_{i} \mid \boldsymbol{\Xi}_{l}\right\rangle \cdot\left\langle\boldsymbol{\Xi}_{l} \mid \boldsymbol{\Xi}_{i}\right\rangle$

i.e., it becomes identical with the left-hand side. The following expression is therefore finally obtained for the Green's function that can be related to the quantum mechanical Bell's experiment:

$$
\begin{aligned}
& \mathbf{G}_{\mathbf{B}}=\sum_{i=1}^{4}\left|\Xi_{i}\right\rangle\left\langle\Xi_{i}\left|\cdot H\left(\left|x_{w}\right|-|x|\right)+\sum_{i, k=1}^{4}[T]_{\mathrm{ik}} \cdot\right| \Psi_{i}\right\rangle\left\langle\Xi_{k}\right| \\
& \cdot H\left(|x|-\left|x_{w}\right|\right) .
\end{aligned}
$$

The probability state of the primary source is on the other hand given by

$|\rho\rangle=\left|\rho_{1}\right\rangle+\left|\rho_{2}\right\rangle$

with

$\left|\rho_{1}\right\rangle=\frac{1}{\sqrt{2}} \cdot\left|\Xi_{2}\right\rangle$

$\left|\rho_{2}\right\rangle=-\frac{1}{\sqrt{2}} \cdot\left|\Xi_{3}\right\rangle$

The probability state (29) with amplitude functions (30) and (31) is then the result of

$\left|\Phi_{\mathrm{QBB}}\right\rangle=\mathbf{G}_{\mathbf{B}}|\rho\rangle$.

If $|x|<\left|x_{w}\right|$ we just get the probability state of the primary source.

Another but not less interesting point of view on the QBB experiment can be provided in the following way: Introducing the normalized probability states

$$
\begin{aligned}
\left|\tilde{\Phi}_{1}\right\rangle:=-\cos \alpha \cdot \sin \beta \cdot\left|\varphi_{1}, \varphi_{1}\right\rangle+\cos \alpha \cdot \cos \beta \\
\quad \cdot\left|\varphi_{1}, \varphi_{2}\right\rangle-\sin \alpha \cdot \sin \beta \cdot\left|\varphi_{2}, \varphi_{1}\right\rangle+\sin \alpha \cdot \cos \beta \cdot\left|\varphi_{2}, \varphi_{2}\right\rangle
\end{aligned}
$$

$\left|\tilde{\Phi}_{2}\right\rangle:=\sin \alpha \cdot \cos \beta \cdot\left|\varphi_{1}, \varphi_{1}\right\rangle+\sin \alpha \cdot \sin \beta$

$$
\cdot\left|\varphi_{1}, \varphi_{2}\right\rangle-\cos \alpha \cdot \cos \beta \cdot\left|\varphi_{2}, \varphi_{1}\right\rangle-\cos \alpha \cdot \sin \beta \cdot\left|\varphi_{2}, \varphi_{2}\right\rangle
$$

$$
\begin{aligned}
& \left|\tilde{\Phi}_{3}\right\rangle:=\frac{1}{\sqrt{2}} \cdot\left[\left|\varphi_{1}, \varphi_{2}\right\rangle+\left|\varphi_{2}, \varphi_{1}\right\rangle\right] \\
& \left|\tilde{\Phi}_{4}\right\rangle:=\frac{1}{\sqrt{2}} \cdot\left[\left|\varphi_{1}, \varphi_{1}\right\rangle+\left|\varphi_{2}, \varphi_{2}\right\rangle\right]
\end{aligned}
$$

will allow us to relate a statistical operator to the QBB experiment. This operator reads

$\hat{\boldsymbol{\rho}}^{(\mathbf{Q B B})}:=\sum_{i=1}^{4} p_{i} \cdot\left|\tilde{\Phi}_{i}\right\rangle\left\langle\tilde{\Phi}_{i}\right|$,

with weights $p_{i}$ given by

$p_{1}=p_{2}=\frac{1}{2}$

$p_{3}=-p_{4}=2 \cdot c(\alpha, \beta)$,

and

$c(\alpha, \beta)=\sin \alpha \cdot \sin \beta \cdot \cos \alpha \cdot \cos \beta$.

$\left|\tilde{\Phi}_{1}\right\rangle$ and $\left|\tilde{\Phi}_{2}\right\rangle$ as well as $\left|\tilde{\Phi}_{3}\right\rangle$ and $\left|\tilde{\Phi}_{4}\right\rangle$ are again orthogonal among each other. Moreover,

$\sum_{i=1}^{4} p_{i}=1$

holds for the weights. Note the negative weight (negative quasi-probability) $p_{4}$ ! The measured probabilities (9) and (10) for any parameter configuration $(\alpha, \beta)$ are then the result of

$$
\begin{aligned}
& p(y, y)=\left\langle\varphi_{1}, \varphi_{1}\left|\hat{\boldsymbol{\rho}}^{(\mathbf{Q B B})}\right| \varphi_{1}, \varphi_{1}\right\rangle \\
& p(n, n)=\left\langle\varphi_{2}, \varphi_{2}\left|\hat{\boldsymbol{\rho}}^{(\mathbf{Q B B})}\right| \varphi_{2}, \varphi_{2}\right\rangle \\
& p(y, n)=\left\langle\varphi_{2}, \varphi_{1}\left|\hat{\boldsymbol{\rho}}^{(\mathbf{Q B B})}\right| \varphi_{1}, \varphi_{2}\right\rangle \\
& p(n, y)=\left\langle\varphi_{1}, \varphi_{2}\left|\hat{\boldsymbol{\rho}}^{(\mathbf{Q B B})}\right| \varphi_{2}, \varphi_{1}\right\rangle,
\end{aligned}
$$

as known from QM. Operator (61) may be called the "basic Bell's operator" since it is related to a single experiment with a fixed parameter configuration $\alpha$ and $\beta$. On the other hand, if we have a classical mixture of $N$ such experiments (for different parameter configurations $\left(\alpha_{k}, \beta_{k}\right)$ with $k=1, \ldots, N)$, and with classical weights $r_{k}, \sum_{k=1}^{N} r_{k}=1$, then we can relate the following statistical operator to the mixture:

$\hat{\mathbf{R}}=\sum_{k=1}^{N} r_{k} \cdot \hat{\boldsymbol{\rho}}_{\mathbf{k}}^{(\mathbf{Q B B})}=\sum_{k=1}^{N} \sum_{i=1}^{4} r_{k} \cdot p_{i}^{(k)} \cdot\left|\tilde{\Phi}_{i}^{(k)}\right\rangle\left\langle\tilde{\Phi}_{i}^{(k)}\right|$.

Regarding the statistical operator (61) the question of the linear independence of the normalized probability states $\left|\tilde{\Phi}_{i}\right\rangle$ is of some importance. If this happens, then we are able to represent any probability state of our 4-dim. event space by a linear combination of these vectors. To prove the linear independence we have to consider Grams matrix

$\mathbf{G}=\left\langle\tilde{\Phi}_{i} \mid \tilde{\Phi}_{j}\right\rangle, \quad i, j=1,2$

Because of (57)-(60) this matrix is a symmetric ones 
$\mathbf{G}=\left(\begin{array}{cccc}1 & 0 & g_{1} & g_{2} \\ 0 & 1 & -g_{1} & g_{2} \\ g_{1} & -g_{1} & 1 & 0 \\ g_{2} & g_{2} & 0 & 1\end{array}\right)$,

with elements $g_{1}$ and $g_{2}$ given by

$g_{1}=\frac{1}{\sqrt{2}} \cdot \cos (\alpha+\beta)$

$g_{2}=\frac{1}{\sqrt{2}} \cdot \sin (\alpha-\beta)$.

Its determinant reads

$\operatorname{det}(\mathbf{G})=\frac{8}{\sqrt{2}} \cdot c(\alpha, \beta)$,

with $c(\alpha, \beta)$ according to (64). Thus we have to meet the condition

$c(\alpha, \beta) \neq 0$

to ensure the linear independence of the vectors (57)-(60). On the other hand, if

$c(\alpha, \beta)=0$

holds (this happens if we have $(\alpha=0$ or $\pi / 2, \beta \neq 0$ ) or $(\alpha \neq 0, \beta=0$ or $\pi / 2)$ ), both weights $p_{3}$ and $p_{4}$ are identical zero. We will see later that the probabilities resulting from the two states $\left|\tilde{\Phi}_{1}\right\rangle$ and $\left|\tilde{\Phi}_{2}\right\rangle$ (i.e., if restricting the summation in (61) to $\sum_{i=1}^{2}$ !) turn out to be the probabilities of the classical counterpart of the QBB experiment. The two states $\left|\tilde{\Phi}_{3}\right\rangle$ and $\left|\tilde{\Phi}_{4}\right\rangle$ can therefore be considered to represent the interference contribution that results from the superposition of the two substates (27) and (28). From (61) we can see moreover that $\left|\tilde{\Phi}_{3}\right\rangle$ acts as a "source state". I.e. it adds a certain amount to the probabilities $p(y, n)$ and $p(n, y)$ calculated from the states $\left|\tilde{\Phi}_{1}\right\rangle$ and $\left|\tilde{\Phi}_{2}\right\rangle$. Contrary, $\left|\tilde{\Phi}_{4}\right\rangle$ acts as a "sink state" since it removes the same amount from the probabilities $p(y, y)$ and $p(n, n)$ calculated from the states $\left|\tilde{\Phi}_{1}\right\rangle$ and $\left|\tilde{\Phi}_{2}\right\rangle$.

\subsection{The CHSH-inequality and its violation with the $Q B B$ experiment}

Now we are prepared to discuss the importance of the CHSH-inequality for the QBB experiment. The following correlation function can be defined using the probabilities related to a fixed parameter set $(\alpha, \beta)$ in our 4-dim. event space:

$C(\alpha, \beta):=p(y, y)+p(n, n)-p(y, n)-p(n, y)$.

It was shown by Clauser et al. [4] that the inequality

$\left|C(\alpha, \beta)-C\left(\alpha, \beta^{\prime}\right)\right|+\left|C\left(\alpha^{\prime}, \beta\right)+C\left(\alpha^{\prime}, \beta^{\prime}\right)\right| \leq 2$

must hold for any experiment that can be described by a theory of local realism. For a moment we will left open what precisely a "theory of local realism" means. This is another aspect of an ongoing discussion about the nature of QM. However, regarding the QBB experiment the measured probabilities (9) and (10) are identical with the probabilities calculated theoretically by use of (66)-(69) with the statistical operator given by (61)-(63). Thus we get the correlation function

$C(\alpha, \beta)=-\cos 2(\alpha-\beta)$.

Then, if we consider the probabilities related the 4 different parameter sets

$$
\begin{aligned}
& (\alpha, \beta)=\left(0, \frac{\pi}{8}\right) \\
& \left(\alpha, \beta^{\prime}\right)=\left(0, \frac{3 \pi}{8}\right) \\
& \left(\alpha^{\prime}, \beta\right)=\left(\frac{\pi}{4}, \frac{\pi}{8}\right) \\
& \left(\alpha^{\prime}, \beta^{\prime}\right)=\left(\frac{\pi}{4}, \frac{3 \pi}{8}\right),
\end{aligned}
$$

inequality (79) is obviously violated. We get $2 \cdot \sqrt{2}$ instead of a value $\leq 2$ ! One may ask: Hey, where is the problem? We have an accepted experimental outcome - the probabilities of the $\mathrm{QBB}$ experiment. And these probabilities result in correlation functions that may violate an inequality for certain parameter configurations. Isn't it simply an indication that there must be something wrong with the derivation of this inequality? Unfortunately, the problem is not that simple. To get a better understanding of its importance and how we have to change the experimental configuration to violate this inequality with a classical optics experiment we want to discuss first the classical counterpart of the above described QBB experiment. Let us call it a "classical Bell's box" experiment (a CBB experiment). A simple but instructive classroom experiment with two sets of differently coloured marbles that fits into the scheme presented in Fig. 3 runs as follows:

A box $B_{p}$ with one white and one black marble represents the primary stochastic source. Two additional boxes $B_{w}$ and $B_{b}$ are filled with 17 white and three black marbles (box $B_{w}$ ), and 17 black and three white marbles (box $B_{b}$ ). This corresponds approximately to the probabilities of $\sin ^{2} 3 \pi / 8=\cos ^{2} \pi / 8 \approx 0.85$ and $\cos ^{2} 3 \pi / 8=\sin ^{2} \pi / 8 \approx$ 0.15 to draw a white or black marble out of the respective box. These two additional boxes represent the local interaction on the right-hand side!

Now, if parameter set (81) is chosen, the experiment is performed in the following way: we draw both marbles blindly out of box $B_{p}$ and put one marble on the left-hand side and the other marble on the right-hand side on our desk. The colour of the marble on the left-hand side is already the result of this side since $\alpha=0$, i.e., since we have no additional interaction on this side. To get the result on the right-hand side requires an additional step. If the primary marble on the right-hand side is white, then we have to draw another marble out of box $B_{w}$. Its colour is the result on the right-hand side. But if the primary marble on the right-hand side is black, then we have to draw another marble out of box $B_{b}$. Its colour will then be the result on the right-hand side. We repeat this procedure until we are able to calculate the probabilities related to the colours of the marbles on both sides within a sufficient accuracy. Then we are able to calculate the correlation function $C(\alpha=0, \beta=\pi / 8)$ according to (78) but with "yes (y)" and 
"no (n)" in the probabilities replaced by "white (w)" and "black (b)").

If parameter set (82) is chosen, the experiment runs as follows: the first step to get the result on the left hand side is as before. But, now, if the primary marble on the righthand side is white, then we have to draw another marble out of box $B_{b}$. Its colour is the result on the right-hand side. On the other hand, if the primary marble on the righthand side is black, then we have to draw another marble out of box $B_{w}$. This colour will then be the result on the right-hand side. We repeat this procedure again until we are able to calculate the probabilities within a sufficient accuracy. Then we are able to calculate the correlation function $C(\alpha=0, \beta=3 \pi / 8)$.

These are the experiments for the two parameter sets (81) and (82). We can proceed in a similar way if the local parameter $\alpha$ on the left-hand side is non-zero. The only thing we have to do is to fill two additional boxes on the left-hand side with an appropriate number of black and white marbles to meet the probabilities of the local interaction on this side. However, since $\alpha=\pi / 4$ for the two sets (83) and (84) $\sin ^{2} \alpha=\cos ^{2} \alpha=1 / 2$ holds. Only one additional box with one white and one black marble is therefore needed in this case on the left-hand side.

Performing this experiment (500 single measurements for a given parameter set $(\alpha, \beta)$ are sufficient to get a quite accurate probability, as me and my family discovered at several rainy weekends) it turns out that the probabilities related to the 4 events $(w, w),(w, b),(b, w)$, and $(b, b)$ (i.e., the colours of the marbles on both sides) are given by

$$
\begin{aligned}
p(w, w)= & p(b, b)=\frac{1}{2} \\
& \cdot\left(\sin ^{2} \alpha \cdot \cos ^{2} \beta+\sin ^{2} \beta \cdot \cos ^{2} \alpha\right) \\
p(w, b)= & p(b, w)=\frac{1}{2} \\
& \cdot\left(\cos ^{2} \alpha \cdot \cos ^{2} \beta+\sin ^{2} \beta \cdot \sin ^{2} \alpha\right) .
\end{aligned}
$$

But these probabilities follow also from the two states (27) and (28) if calculating the probabilities from each state separately, and by adding the corresponding probabilities afterwards. Alternatively, we can restrict the summation in the statistical operator (61) to $\sum_{i=1}^{2}$, as already mentioned. Once we accept these probabilities as an experimental fact of the CBB experiment, from (78) we can calculate the corresponding correlation function to get

$C(\alpha, \beta)=-\cos 2 \alpha \cdot \cos 2 \beta$.

But with this correlation function we would never be able to violate the $\mathrm{CHSH}$-inequality (79). The proof runs as follows:

Due to definition (78)

$|C(\alpha, \beta)| \leq 1$

holds for any parameter set $(\alpha, \beta)$. It holds also if the probabilities in (78) are replaced by corresponding normalized intensities. That is exactly what we intend to do in the classical optics experiment that is described in the second part of this paper. Next, let us express the difference between the two functions $C(\alpha, \beta)$ and $C\left(\alpha, \beta^{\prime}\right)$ by

$$
\begin{aligned}
& C(\alpha, \beta)-C\left(\alpha, \beta^{\prime}\right)=C(\alpha, \beta) \cdot\left[1 \pm C\left(\alpha^{\prime}, \beta^{\prime}\right)\right]-C\left(\alpha, \beta^{\prime}\right) \\
& \quad \cdot\left[1 \pm C\left(\alpha^{\prime}, \beta\right)\right]=C(\alpha, \beta)-C\left(\alpha, \beta^{\prime}\right) \\
& \quad \pm\left[C(\alpha, \beta) \cdot C\left(\alpha^{\prime}, \beta^{\prime}\right)-C\left(\alpha, \beta^{\prime}\right) \cdot C\left(\alpha^{\prime}, \beta\right)\right] .
\end{aligned}
$$

This is obviously an identity only if

$C(\alpha, \beta) \cdot C\left(\alpha^{\prime}, \beta^{\prime}\right)-C\left(\alpha, \beta^{\prime}\right) \cdot C\left(\alpha^{\prime}, \beta\right)=0$.

I.e., if this condition holds (89) represents nothing but adding a "nutritious zero" to the difference of $C(\alpha, \beta)$ and $C\left(\alpha, \beta^{\prime}\right)$. Taking the inequality

$|a-b| \leq|a|+|b|$

into account we thus get from (89)

$$
\begin{gathered}
\left|C(\alpha, \beta)-C\left(\alpha, \beta^{\prime}\right)\right| \leq\left|C(\alpha, \beta) \cdot\left[1 \pm C\left(\alpha^{\prime}, \beta^{\prime}\right)\right]\right|+\mid C\left(\alpha, \beta^{\prime}\right) \\
\cdot\left[1 \pm C\left(\alpha^{\prime}, \beta\right)\right] \mid .
\end{gathered}
$$

The expressions inside the brackets on the right-hand side are always $\geq 0$, according to (88). Therefore we can also write

$$
\begin{gathered}
\left|C(\alpha, \beta)-C\left(\alpha, \beta^{\prime}\right)\right| \leq|C(\alpha, \beta)| \cdot\left[1 \pm C\left(\alpha^{\prime}, \beta^{\prime}\right)\right]+\left|C\left(\alpha, \beta^{\prime}\right)\right| \\
\cdot\left[1 \pm C\left(\alpha^{\prime}, \beta\right)\right] .
\end{gathered}
$$

From this expression and from (88) the inequality

$$
\begin{aligned}
\left|C(\alpha, \beta)-C\left(\alpha, \beta^{\prime}\right)\right| & \leq 2 \pm\left[C\left(\alpha^{\prime}, \beta^{\prime}\right)+C\left(\alpha^{\prime}, \beta\right)\right] \\
& \leq 2-\left|C\left(\alpha^{\prime}, \beta^{\prime}\right)+C\left(\alpha^{\prime}, \beta\right)\right|
\end{aligned}
$$

follows in a straightforward way. Now it becomes clear that our $\mathrm{CBB}$ experiment can never violate the $\mathrm{CHSH}-$ inequality since condition (90) holds for its correlation function (87). But the correlation function (80) of the QBB experiment clearly violates condition (90). Especially the 4 parameter sets (81)-(84) result in a maximum violation. Thus we have found the necessary condition

$C(\alpha, \beta) \cdot C\left(\alpha^{\prime}, \beta^{\prime}\right)-C\left(\alpha, \beta^{\prime}\right) \cdot C\left(\alpha^{\prime}, \beta\right) \neq 0$

to violate the $\mathrm{CHSH}$-inequality. To emphasize the essential difference between the QBB- and CBB experiment I want to state once again that the former experiment requires the superposition of the two states (27) and (28) before calculating the probabilities. This superposition provides an additional interference contribution that is already known from the quantum mechanical double-slit experiment. Regarding this experiment we get the typical interference pattern of the double-slit even if sending the quantum particles (photons or electrons, see [12] for example) one after another through the double-slit. Contrary, in the CBB experiment the probabilities are calculated separately from each state and added afterwards. Thus we have a further confirmation of Feynman's statement: "I will take just this one experiment, which has been designed to contain all of the mystery of quantum mechanics, to put you up against the paradoxes and mysteries and peculiarities of nature one hundred per cent. Any other situation in quantum mechanics, it turns out, can always be explained by saying: You remember the case of the experiment with the two holes? It's the same thing" [13]. But it is not the superposition itself that seems a mystery from our classical lines of thought. Superposition is already a known 
effect from classical field theories. It's rather the aspect that we have to superpose two abstract probability states that belong to different situations in our QBB experiment which do not exist at the same time. Contrary, the superposition of two classical fields necessarily requires the existence of the fields in a certain volume of space at the same time.

Now, let us see how we have to modify the scheme of the 4-dim. event space considered so far to violate the $\mathrm{CHSH}$-inequality with a classical optics experiment.

\section{Violation of the CHSH-Inequality with a classical optics experiment}

3.1. Modification of the 4-dim. event space related to Bell's experiment

Fig. 4 shows the modification of the scheme of Bell's experiment represented in Fig. 3. We will first discuss the upper branch of Fig. 4. A primary source generates a linearly polarized plane wave with amplitude $E_{0}$. After the first interaction $W_{1}(\alpha)$ a part of this plane wave propagates horizontally polarized and with amplitudes given in the square brackets along the two directions $a^{\prime}$ and $b^{\prime}$. After the second interaction $W_{2}(\beta)$ we have a horizontally and a vertically polarized part along both directions with amplitudes given in the square brackets. The coherent superposition of the respective parts will allow us to measure the two intensities $I_{h h}$ and $I_{h v}$. The same procedure holds for the lower branch of Fig. 4 except that we have a vertically polarized plane wave after the first interaction. In this way we will be able to measure the two intensities $I_{v h}$ and $I_{v v}$ after the second interaction. These 4 intensities are now the measurement values of a 4-dim. event space. We may represent the finally superposed

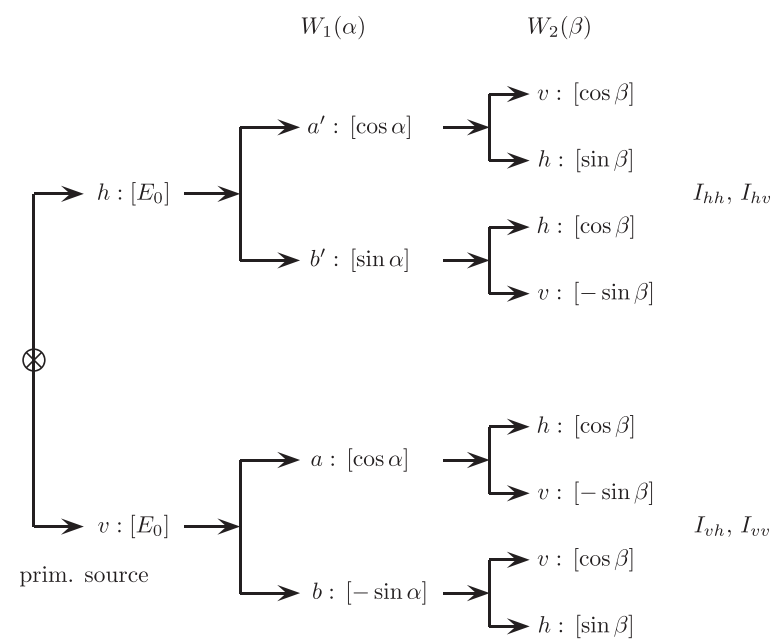

Fig. 4. Scheme of a classical optics experiment with two successive interactions $W_{1}(\alpha)$ and $W_{2}(\beta)$. The horizontally and vertically polarized subfields after the interactions are coherently superposed, and the intensities $I_{h h}, I_{h v}, I_{v h}$, and $I_{v v}$ are measured afterwards. fields in a corresponding direct product space by

$$
\begin{aligned}
& \left|\Phi_{1}\right\rangle=E_{0} \\
& \quad \cdot\left[\cos (\alpha-\beta) \cdot\left|\varphi_{2}, \varphi_{1}\right\rangle+\sin (\alpha-\beta) \cdot\left|\varphi_{2}, \varphi_{2}\right\rangle\right]
\end{aligned}
$$

and

$$
\begin{aligned}
& \left|\Phi_{2}\right\rangle=E_{0} \\
& \quad \cdot\left[\sin (\alpha-\beta) \cdot\left|\varphi_{1}, \varphi_{1}\right\rangle+\cos (\alpha-\beta) \cdot\left|\varphi_{1}, \varphi_{2}\right\rangle\right] .
\end{aligned}
$$

$\left|\varphi_{1}, \varphi_{1}\right\rangle$ is the basis vector that represents the vertically polarized state of the field after the second interaction if we have a vertically polarized part after the first interaction. Correspondingly, $\left|\varphi_{1}, \varphi_{2}\right\rangle$ is the basis vector that represents the horizontally polarized state of the field after the second interaction if we have a vertically polarized part after the first interaction, etc. Thus we obtain the intensities

$I_{h h}=I_{v v}=E_{0}^{2} \cdot \sin ^{2}(\alpha-\beta)$

$I_{h v}=I_{v h}=E_{0}^{2} \cdot \cos ^{2}(\alpha-\beta)$,

from the scalar products of the projections of (96) and (97), as demonstrated in Section 2.1. If calculating the correlation function according to

$C(\alpha, \beta)=\frac{1}{I_{t}} \cdot\left(I_{h h}+I_{v v}-I_{h v}-I_{v h}\right)$

with the total intensity $I_{t}$ given by

$I_{t}=I_{h h}+I_{h v}+I_{v h}+I_{v v}=E_{0}^{2} \cdot \cos ^{2}(\alpha-\beta)$

we end up with

$C(\alpha, \beta)=-\cos 2(\alpha-\beta)$.

This correlation function results again in a maximum violation of the $\mathrm{CHSH}$-inequality if the four different parameter configurations (81)-(84) are considered.

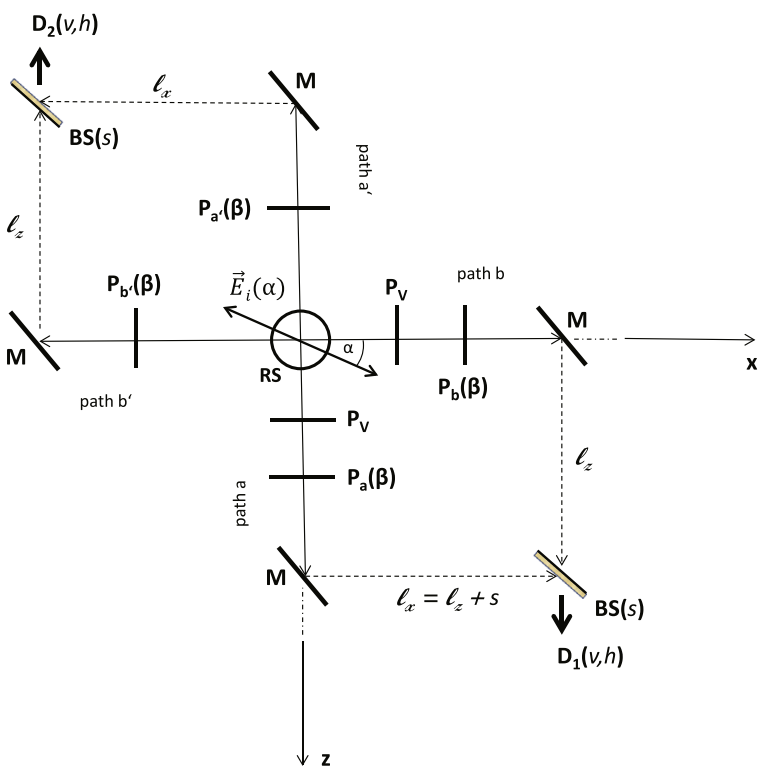

Fig. 5. Classical optics experiment related to the scheme of Fig. 4. It consists of a Rayleigh scatterer that combines two Mach-Zehnder interferometers. 
3.2. A combination of Rayleigh scattering with a MachZehnder interferometry

How can we put a corresponding experiment into practice? A possible configuration is shown in Fig. 5. It is a combination of a Rayleigh scattering process with two Mach-Zehnder interferometers. A detailed description of the phase dependencies in the proposed MachZehnder setup can be found in [14], for example. The first interaction process, i.e., the Rayleigh scattering process (RS), depends on the parameter $\alpha$. Within the Mach-Zehnder setup we have a second interaction process by differently rotating the plane of polarization of the subfields along each path. But all 4 rotations are characterized by the same parameter $\beta$. We will denote a linearly polarized plane wave as "horizontally polarized" if its $E$-vector is oscillating in the $x-z$-plane, and "vertically polarized" if it is oscillating perpendicularly to this plane, i.e., if it is oscillating in $y$-direction. The RS is located at the origin of the coordinate system. A linearly polarized plane wave $\vec{E}_{i}(\alpha)$ propagates along the positive $y$-axis and impinges on the RS. Its plane of polarization forms an angle of $\alpha$ with the $x$-axis. According to Rayleigh scattering (see, e.g., [15]) we then have the following two components of linearly polarized fields traveling along the positive and negative $x$-direction (i.e. traveling along path $b$ and $b^{\prime}$ ):

- path b:

$$
\left|\psi_{b}\right\rangle=E_{0} \cdot \sin \alpha \cdot|\hat{z}\rangle
$$

- path $b^{\prime}$ :

$$
\left|\psi_{b^{\prime}}\right\rangle=E_{0} \cdot \sin \alpha \cdot|\hat{z}\rangle \text {. }
$$

On the other hand, the components of the fields traveling along the positive and negative $z$-direction (i.e. traveling along path $a$ and $a^{\prime}$ ) are given by

- path a:

$$
\left|\psi_{a}\right\rangle=E_{0} \cdot \cos \alpha \cdot|\hat{x}\rangle
$$

- path $a^{\prime}$ :

$$
\left|\psi_{a^{\prime}}\right\rangle=E_{0} \cdot \cos \alpha \cdot|\hat{x}\rangle
$$

with $|\hat{x}\rangle$ and $|\hat{z}\rangle$ being the unit vectors in $x$ - and $z$-directions. After changing the horizontal polarization along path $a$ and $b$ into a vertical polarization by the two halfwave plates $\mathbf{P}_{\mathbf{V}}$ we have

- path b:

$$
\left|\psi_{b}\right\rangle=E_{0} \cdot \sin \alpha \cdot|\hat{y}\rangle
$$

- path a:

$$
\left|\psi_{a}\right\rangle=E_{0} \cdot \cos \alpha \cdot|\hat{y}\rangle \text {. }
$$

Regarding the polarization along path $a, b, a^{\prime}$ and $b^{\prime}$ we are now in a state that corresponds to the $\alpha$-dependent splitting after the first interaction $W_{1}(\alpha)$ of Fig. 4 .

Next, let us proceed with path $a$ and $b$. With the two half-wave plates $\mathbf{P}_{\mathbf{a}}(\boldsymbol{\beta})$ and $\mathbf{P}_{\mathbf{b}}(\boldsymbol{\beta})$ the vertical polarization of each subfield is again rotated in such a way, that the superposition of

- path a:

$$
\begin{aligned}
\left|\psi_{a}\right\rangle=- & E_{0} \cdot \cos \alpha \cdot \sin \beta \cdot|\hat{y}\rangle \\
& +E_{0} \cdot \cos \alpha \cdot \cos \beta \cdot|\hat{x}\rangle
\end{aligned}
$$

- path b:

$$
\begin{aligned}
\left|\psi_{b}\right\rangle= & E_{0} \cdot \sin \alpha \cdot \cos \beta \cdot|\hat{y}\rangle \\
& +E_{0} \cdot \sin \alpha \cdot \sin \beta \cdot|\hat{x}\rangle
\end{aligned}
$$

can be accomplished in the lower beam splitter BS but into the direction of detector $\mathbf{D}_{\mathbf{1}}$. To this end, corresponding phase shifts by $\pi$, that may caused by the beam splitter as well as by the reflecting mirrors, have to be considered in the rotation of the plane of polarization of each subfield along path a and b. Then, with detector $\mathbf{D}_{1}(\mathbf{v}, \mathbf{h})$, we are able to measure the intensities

$I_{v h}=E_{0}^{2} \cdot \cos ^{2}(\alpha-\beta)$

and

$I_{v v}=E_{0}^{2} \cdot \sin ^{2}(\alpha-\beta)$

of the vertically and horizontally polarized components of the superposition.

The behaviour along path $b^{\prime}$ and $a^{\prime}$ can be described in a similar manner with the difference that we have already horizontally polarized subfields as the initial states. The plane of polarization of these two subfields are then rotated with the two half-wave plates $\mathbf{P}_{\mathbf{a}^{\prime}}(\boldsymbol{\beta})$ and $\mathbf{P}_{\mathbf{b}^{\prime}}(\boldsymbol{\beta})$ in such a way, that the superposition of

- path $a^{\prime}$ :

$$
\begin{aligned}
\left|\psi_{a^{\prime}}\right\rangle= & E_{0} \cdot \cos \alpha \cdot \cos \beta \cdot|\hat{y}\rangle \\
& -E_{0} \cdot \cos \alpha \cdot \sin \beta \cdot|\hat{x}\rangle
\end{aligned}
$$

- path $b^{\prime}$ :

$$
\begin{aligned}
\left|\psi_{b^{\prime}}\right\rangle= & E_{0} \cdot \sin \alpha \cdot \sin \beta \cdot|\hat{y}\rangle \\
& +E_{0} \cdot \sin \alpha \cdot \cos \beta \cdot|\hat{x}\rangle
\end{aligned}
$$

can be accomplished in the upper beam splitter BS but into the direction of detector $\mathbf{D}_{\mathbf{2}}$. The corresponding phase shifts by $\pi$, that may caused by the beam splitter as well as by the reflecting mirrors in the upper Mach-Zehnder interferometer, must again be taken into account in the rotation of the plane of polarization of each subfield along path $a^{\prime}$ and $b^{\prime}$. Then, with detector $\mathbf{D}_{\mathbf{2}}(\mathbf{v}, \mathbf{h})$, we are able to 
measure the intensities

$I_{h v}=E_{0}^{2} \cdot \cos ^{2}(\alpha-\beta)$,

and

$I_{h h}=E_{0}^{2} \cdot \sin ^{2}(\alpha-\beta)$

of the vertically and horizontally polarized components of the superposition. Finally, calculating the correlation function from these four intensities according to (100) and (101) provides us with expression (102). However, if we measure these four intensities right after the four halfwave plates $\mathbf{P}_{\mathbf{a}}(\boldsymbol{\beta}), \mathbf{P}_{\mathbf{b}}(\boldsymbol{\beta}), \mathbf{P}_{\mathbf{a}^{\prime}}(\boldsymbol{\beta})$, and $\mathbf{P}_{\mathbf{b}^{\prime}}(\boldsymbol{\beta})$, then, after normalization, we would end up with intensities that are identical with the classical probabilities (85) and (86). It should be also mentioned that due to the normalization of the finally measured intensities, the reflecting mirrors in the two Mach-Zehnder interferometers may be replaced by unpolarizing beam splitters. But all these beam splitters must be characterized by the same ratio of the reflected and transmitted intensities.

\section{Conclusion}

An abstract probability state description in a 4-dim. direct product space was introduced to discuss the difference between a typical quantum mechanical Bell's experiment and its classical counterpart. This difference could be traced back to the superposition of two substates before the calculation of the related probabilities in the former experiment. Contrary, the probabilities of its classical counterpart must be calculated from each substate separately and added afterwards. A T-matrix and a Green's function as well as a statistical operator can be defined in this 4-dim. product space that allow the description of both experiments from different point of views. The negative weight or quasi-probability of the statistical operator acts as a sink of probabilities related to two of the single events in the considered 4-dim. event space. Moreover, a necessary condition to violate the $\mathrm{CHSH}-$ inequality was derived and applied to both experiments. Thus it was proven that the $\mathrm{CHSH}$-inequality can never be violated by the classical counterpart of the quantum mechanical Bell's experiment. It was then shown that the violation of the $\mathrm{CHSH}$-inequality with a classical optics experiment requires a modification of the 4-dim. event space, and that this modification may be put into practice with a Rayleigh scattering process that connects two Mach-Zehnder interferometers.

\section{References}

[1] Bell JS. Physics 1964;1:195.

[2] Einstein A, Podolsky B, Rosen N. Phys Rev 1935;47:777.

[3] Bohr N. Phys Rev 1935;48:696.

[4] Clauser JF, Horne MA, Shimony A, Holt RA. Phys Rev Lett 1969;23: 880.

[5] Aspect A, Grangier P, Roger G. Phys Rev Lett 1982;49:1804.

[6] Brunner N, et al. arXiv:1303.2849; 2013.

[7] Hemmick DL, Shakur AM. Bell's theorem and quantum realism. Berlin, Heidelberg: Springer; 2012.

[8] Borges CVS, et al. Phys Rev A 2010;82:033833.

[9] Feynman RP. Quantum implications: essays in honour of David Bohm. London, New York: Routledge and Kegan Paul Ltd.; 1987.

[10] Burgin M. arXiv:1008.1287; 2010.

[11] Rother T, Kahnert M. Electromagnetic wave scattering on nonspherical particles. Berlin, Heidelberg: Springer; 2014.

[12] Menzel R, et al. J Mod Opt 2012. http://dx.doi.org/10.1080/ 09500340.2012.746400.

[13] Feynman RP. The character of physical law. Cambridge, MA and London: M.I.T Press; 1967.

[14] Zetie KP, Adams SF, Tocknell RM. Phys Educ 2000;35(1):46.

[15] van de Hulst HC. Light scattering by small. New York: Particles Dover Publ.; 1981. 